\title{
Resonant and nonresonant processes in attosecond streaking from metals
}

\author{
A. G. Borisov, ${ }^{1}$ D. Sánchez-Portal, ${ }^{2}$ A. K. Kazansky, ${ }^{2,3,4}$ and P. M. Echenique ${ }^{2,3,5}$ \\ ${ }_{1}^{1}$ ISMO, UMR 8214 CNRS-Université Paris-Sud, Bâtiment 351, Université Paris-Sud, 91405 Orsay Cedex, France \\ ${ }^{2}$ Centro de Física de Materiales CFM - Materials Physics Center MPC, Centro Mixto CSIC-UPV/EHU, 20018 San Sebastián/Donostia, Spain \\ ${ }^{3}$ Donostia International Physics Center (DIPC), Paseo de Manuel Lardizabal 4, 20018 San Sebastián/Donostia, Spain \\ ${ }^{4}$ IKERBASQUE, Basque Foundation for Science, 48011 Bilbao, Spain \\ ${ }^{5}$ Departamento de Física de Materiales, Facultad de Ciencias Químicas, Universidad del País Vasco UPVIEHU, Apartado 1072, \\ 20080 San Sebastián/Donostia, Spain \\ (Received 22 December 2012; published 22 March 2013)
}

\begin{abstract}
We report on the theoretical study of laser-assisted attosecond photoemission from metals. The full timedependent quantum approach reveals the role of the resonant interband and nonresonant surface emission processes in formation of final atto-streaking spectra. The present results explain recent experimental data on magnesium and show that the valence band streaking essentially reflects the respective weight of surface and resonant bulk electron ejection.
\end{abstract}

DOI: 10.1103/PhysRevB.87.121110

PACS number(s): 78.47.J-, 68.35.Ja, 78.66.Bz, 79.60.-i

The development of attosecond laser pulses ${ }^{1}$ allowed probing the ultrafast electron dynamics in various systems on the atomic time scale ${ }^{2-4}$ (the atomic unit of time is about 24 as). In particular, the attosecond streaking spectroscopy $(\mathrm{ASS})^{5-7}$ has enabled observation of the decay of an inner-shell vacancy in isolated atoms ${ }^{8}$ and determination of the delay in electron photoemission. ${ }^{9-11}$ The ASS technique is based on combination of inherently synchronized powerful nearinfrared (NIR) pulse and the attosecond extreme-ultraviolet (XUV) pulse. The time of the electron ejection from the target by XUV photons is transposed by the NIR field into the electron energy spectrum.

While spectacular progress has been reached in the gas phase, only a few experimental ASS studies have been performed so far for solids. ${ }^{12,13}$ This is despite that a wealth of many-body processes in solids evolve on the attosecond time scale and can be a priori addressed. ${ }^{14-19}$ The first experimental application of ASS to the $\mathrm{W}(110)$ metal surface ${ }^{12}$ has shown that the electrons photoexcited from delocalized valence band (VB) states are ejected approximately 100 as prior to those from localized $4 f$ states (LS). This finding was reproduced in a number of theoretical studies. ${ }^{12,20-22}$ However, complex band structure and screening properties of tungsten ${ }^{12,21,23,24}$ resulted in substantially different model descriptions of the atto-streaking experiment so that the interpretation of the results is controversial.

In this respect a very recent ASS experiment ${ }^{13}$ on $\operatorname{Mg}(0001)$ allows approaching experimental and theoretical objects of study. Indeed, magnesium is a prototype of a free-electron metal; ${ }^{25-27}$ i.e., the model used in full quantum treatments ${ }^{20,22,28}$ should apply. For the free-electron metal the screening of the NIR field develops within the surface layer. ${ }^{29}$ Then, for the LS photoemission, the overall time delay between electron ejection from the $2 p$ core orbital of $\mathrm{Mg}$ and electron entrance into the streaking field above the surface is $\tau \approx \lambda / v$, where $v$ is the free-electron speed. The inelastic mean-free path (MFP) $\lambda$ is of order one to two lattice constants in the relevant energy range. As to the VB emission, the quantum results ${ }^{20,28}$ have shown that the corresponding electrons are excited at the surface and quasi-instantaneously enter the streaking field. Thus, similar to $\mathrm{W}(110)$, the LS/VB emission delay should be typically in 100 as range. This is in sheer contrast with experiment on $\operatorname{Mg}(0001)$ showing negligible time delay between the LS and the VB electrons consistent with the bulk origin of both. ${ }^{13}$

In this work we lift the contradiction between the calculations and experiment on $\mathrm{Mg}$. Our study bridges conventional theory of photoemission ${ }^{30}$ and dynamics of attosecond electron ejection from solids. We show that the time delay in VB electron ejection from solids is determined by two simultaneous contributions to the ionization yield: the nonresonant surface emission and bulk emission due the resonant interband transitions at fixed Bloch momentum of the electron k. We reproduce the experimental data on $\mathrm{Mg}$ and explain the difference between the experimental results on $\mathrm{Mg}$ and $\mathrm{W}$.

We address the experimental case of the photoemission along the surface normal ${ }^{12,13}$ and thus consider a 1D model of $\operatorname{Mg}(0001)$ with potential depending only on the coordinate $z$ perpendicular to the surface. The details of the calculation of the streaking spectra from metals are given elsewhere, ${ }^{20}$ so we only discuss the aspects specific for the present study. In brief, the time-dependent Schrödinger equation for the electron wave packet $\Psi(z, t)$ ejected from initial state $\Phi_{i}(z)$ is formulated in the rotating wave approximation for the weak XUV pulse (atomic units are used unless otherwise stated)

$$
\begin{aligned}
i \partial \Psi(z, t) / \partial t= & \left(H-\Omega_{X}-E_{i}\right) \Psi(z, t) \\
& +\epsilon_{L}(z, t) \Psi(z, t)-i A_{X}(t) \partial \Phi_{i}(z) / \partial z .
\end{aligned}
$$

Equation (1) is directly solved on the equidistant mesh in real space. $\Omega_{X}$ is the carrier frequency of the XUV pulse, and $E_{i}$ is the energy of the initial state. The last term describes interaction of the initial state with XUV pulse in velocity gauge so that the Fourier grid pseudospectral technique ${ }^{31}$ can be implemented for time propagation. We use a Gaussian envelope of the vector potential $A_{X}(t)$ corresponding to the XUV pulse duration of 750 as, similar results have been obtained with 435 as XUV pulse. The $\epsilon_{L}(z, t)$ describes the NIR pulse treated in the length gauge to allow for a numerically efficient account of the IR screening below the surface image plane. The frequency of the $10 \mathrm{fs}$ NIR pulse is set to $1.6 \mathrm{eV}$, 

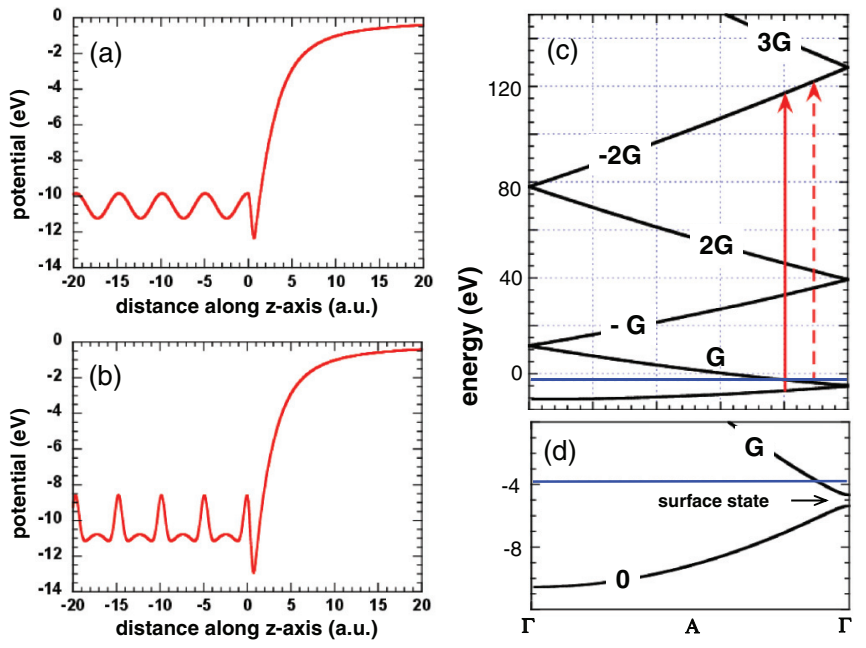

FIG. 1. (Color online) Model potentials $U_{1}(z)$ (a) and $U_{2}(z)$ (b) as functions of the electron coordinate $z$ measured with respect to the last atomic plane. Panel (c) shows the band structure which is graphically undistinguishable for two potentials. Bands are labeled according to reciprocal vector exchange for the resonant process from the lowest band. $G=2 \pi / a$ is the primitive reciprocal lattice vector, and $a=4.923 a_{0}$ is the spacing between atomic planes (Ref. 32). Arrows indicate the resonant transitions at $\Omega_{X}=125 \mathrm{eV}$. Panel (d) zooms into the structure of low-energy bands. Horizontal lines in (c) and (d) denote the Fermi level.

and the intensity of the NIR laser field is $10^{11} \mathrm{~W} / \mathrm{cm}^{2}$. (For further details see Ref. 20.)

$$
H=-\frac{1}{2} \frac{\partial^{2}}{\partial z^{2}}+U(z)+V_{e-h}(z)-i \gamma(z)
$$

is the Hamiltonian of the electron-metal system without laser fields. The absorbing potential $i \gamma(z)$ accounts for the inelastic effects in the bulk. For the ejection of the LS $2 p$ electron the interaction between the electron and the core hole $V_{e-h}(z)$ is given by the screened Yukawa potential. This term is not present for the VB emission.

The most relevant for the present discussion is the choice of the potential $U(z)$ for electron-metal interaction. For the periodic potential inside the solid the one-electron optical transitions are only possible under preservation of the electron Bloch momentum $k_{z} \cdot{ }^{30}$ For the delocalized VB states one then expects (i) an emission from the surface region, where the periodicity is broken and the potential changes from the metal to the vacuum one; (ii) an emission from the inside bulk via the resonant interband transition, where an initial state with energy $E_{i}\left(k_{z}\right)$ is coupled to the final state with energy $E_{f}\left(k_{z}\right)$ such that $E_{f}\left(k_{z}\right)=E_{i}\left(k_{z}\right)+\Omega_{X}$. In Fig. 1 the resonant processes correspond to the vertical transitions shown with arrows.

To shed light on the role of surface and resonant bulk emission of the VB electrons in the final streaking spectra, we have performed calculations with two model potentials $U_{1,2}(z)$ shown in Fig. 1. First, $U_{1}(z)$ is the analytical potential derived by Chulkov et al. ${ }^{32}$ in such a way that the projected band structure of $\mathrm{Mg}(0001)$ is well reproduced: band gap extending from -1.7 to $-1 \mathrm{eV}$, and surface state at $-1.5 \mathrm{eV}$ with respect to the Fermi level [the work function of $\mathrm{Mg}(0001)$ is $3.66 \mathrm{eV}$ ]. ${ }^{32}$ Essentially, the $-1 / 4 z$ image potential tail in vacuum is joined

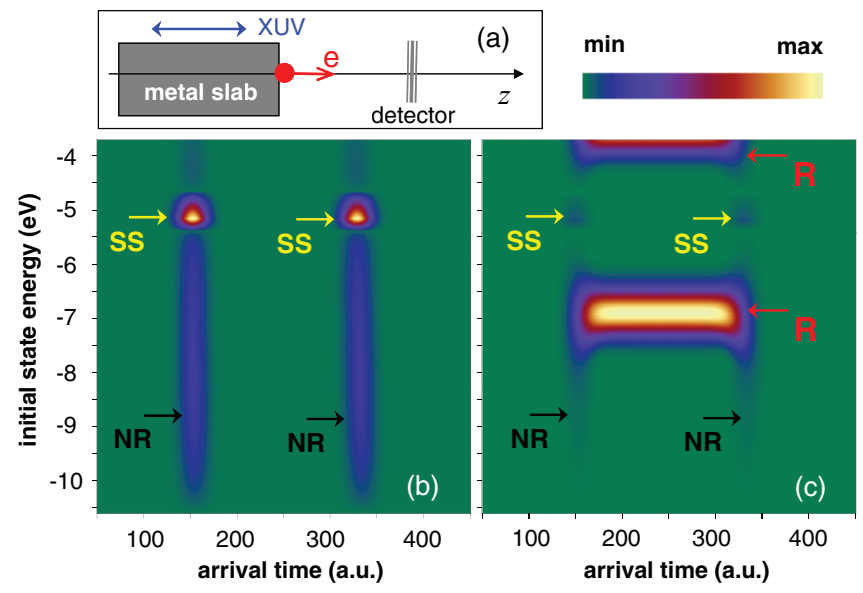

FIG. 2. (Color online) (a) Sketch of the numerical experiment, and a signal on detector obtained with potential $U_{1}(z)$ (b) and $U_{2}(z)$ (c). Results are shown as a function of the energy of the initial state and time after the XUV flash. Nonresonant surface emission (NR), emission from the surface state (SS), and resonant emission (R) are labeled.

smoothly with periodic $\cos (2 \pi z / a)$ function in the bulk. The second potential $U_{2}(z)$ mimics the orthogonality constraint with respect to the core electrons. It is constructed from $U_{1}(z)$ by adding an $\alpha \exp \left[-\left(z-z_{n}\right)^{2} /(0.12 a)^{2}\right](\alpha=3 \mathrm{eV})$ term at each atomic plane located at $z_{n}$. Correction is then applied such that zero and first harmonics of $U_{2}(z)$ are equal to that of $U_{1}(z)$. Thus, the projected band structures obtained with both potentials are nearly the same (see Fig. 1).

We have first calculated the initial state resolved photoemission from the 110 layers thick $\operatorname{Mg}(0001)$ slab by $\Omega_{X}=125 \mathrm{eV}$ XUV pulse incident at time $t=0$. For transparent discussion of the role of different emission regimes, the absorbing optical $\gamma(z)$ and the NIR field are set to zero. The signal $\left|\Psi\left(z_{d}, t\right)\right|^{2}$ obtained on the "detector" located at $z_{d}=240 a_{0}$ above the surface is shown in Fig. 2 as a function of time and the energy $E_{i}$ of the initial occupied VB state subjected to photoionization. The energies $E_{i}$ span the interval from the bottom of the VB to the Fermi level so that all occupied VB states have been considered.

The results obtained with two model potentials are drastically different. This is because the $U_{2}(z)$ potential contains enough high harmonics to trigger noticeable resonant interband transition in agreement with synchrotron photoemission studies of $\operatorname{Mg}(0001)^{25}$ while the $U_{1}(z)$ potential is too soft. In the $U_{1}(z)$ case the electrons are preferentially ejected by the nonresonant process from the surfaces of the metal slab. The difference of the arrival time is given by the slab thickness. Photoemission from the surface states appears as bright spots at $-5.2 \mathrm{eV}$. In the $U_{2}(z)$ case the signal is dominated by the resonant contributions with electrons coming from the metal bulk due to the resonant process. In this case, the entire slab appears as a source for electron emission. The two resonances correspond to transitions from the occupied (0) and partially occupied $(G)$ band to the $(-2 G)$ band of the excited states as shown in Fig. 1. The $E_{i}$ width of the resonances reflects the finite duration of the XUV pulse.

Thus, the resonant interband process leads to VB electron emission from inside metal similar to the LS electron emission. 

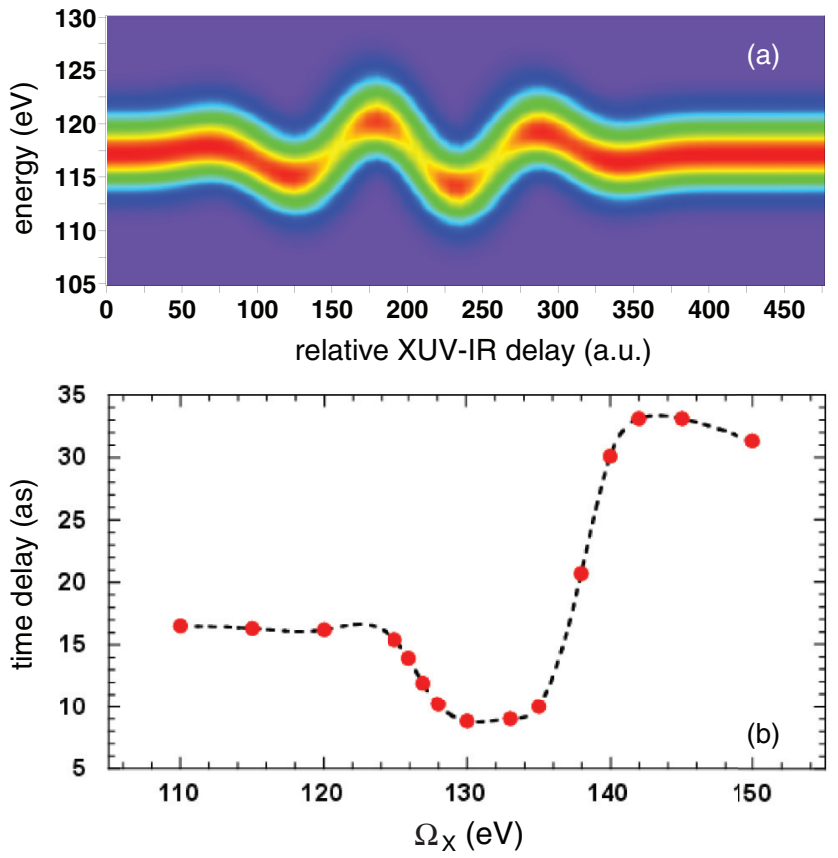

FIG. 3. (Color online) (a) Attosecond time-resolved photoemission spectrum calculated for VB with $U_{2}(z)$ model potential and $\Omega_{X}=125 \mathrm{eV}$. (b) Calculated relative delay between the VB and LS $2 p$ electron emission obtained from COEs of the streaking spectra. Results are shown as a function of the XUV energy. Positive delay implies that VB electrons are first to leave the metal.

The relative delay $\Delta$ in VB and LS streaking can be then estimated from

$$
\Delta=\tau_{L S}-\tau_{V B} \approx \frac{\lambda_{\mathrm{LS}}}{\sqrt{2 E_{\mathrm{LS}}}}-(1-\mathcal{P}) \frac{\lambda_{\mathrm{VB}}}{\sqrt{2 E_{\mathrm{VB}}}},
$$

where $\lambda_{\mathrm{LS}}\left(\lambda_{\mathrm{VB}}\right)$ is the mean free path ${ }^{33}$ of the electron ejected from LS $(\mathrm{VB}), \tau_{\mathrm{LS}}\left(\tau_{\mathrm{VB}}\right)$ is the LS (VB) emission delay, $E_{\mathrm{LS}}$ $\left(E_{\mathrm{VB}}\right)$ is the corresponding final-state energy, and $\mathcal{P}$ stands for the weight of the surface emission in the VB signal. Here we assume that the electrons ejected from the surface enter the streaking field without delay. For $\mathcal{P}=0$ (interband transition only) Eq. (3) leads to $\tau_{\mathrm{LS}} \approx \tau_{\mathrm{VB}} \approx 85$ as and $\Delta \approx 0$ in conditions of $\mathrm{Mg}(0001)$ experiment as has been pointed out in Ref. 13.

Full numerical treatment of the VB and LS streaking confirms the importance of the resonant process for the extracted photoemission delay. In Fig. 3(a) we show the typical streaking spectra of the VB emission obtained by summing contributions from all possible initial states. The center of energy $(\mathrm{COE})$ of the streaking spectra is then used to define the relative delay between LS $2 p$ and VB electrons. ${ }^{13,20}$ The final results for $\Delta$ obtained including the resonant process are shown in Fig. 3(b) as a function of the energy of XUV photons $\Omega_{X}$. We obtained that $\tau_{\mathrm{LS}}$ is nearly constant over the studied $\Omega_{X}$ range. Therefore the variation of the relative delay $\Delta$ is due to the variation of the VB emission delay $\tau_{\mathrm{VB}}$. For $110 \leqslant \Omega_{X} \leqslant 120$ $\mathrm{eV}$ the bulk emission proceeds via the $(0):(-2 G)$ resonance (see Fig. 1). The $2 p$ electrons are delayed from the VB electrons by $\Delta \approx 16$ as. Thus, the theoretical result agrees with reported experimental value of $5 \pm 20$ as. ${ }^{13}$ Calculations performed with $U_{1}(z)$ model potential give $\Delta \approx 60$ as roughly

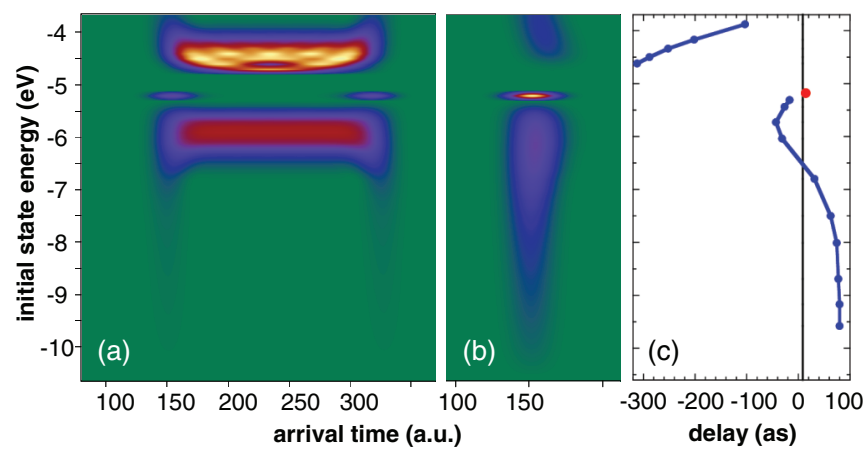

FIG. 4. (Color online) Signal on detector obtained for $\Omega_{X}=$ $130 \mathrm{eV}$ with potential $U_{2}(z)$ in the absence of the NIR field, (a) without and (b) with account for the MFP inside metal. Results are shown as a function of the energy of the initial state and time after the XUV flash. (c) Initial VB state resolved delay between the VB and LS $2 p$ electron emission. Positive delay implies VB electrons ejected first. The vertical line at 9 as shows the average delay obtained from the COEs of the LS and VB streaking spectrograms. Red dot shows the surface-state data.

consistent with Eq. (3) for $\mathcal{P}=1$. This result is also consistent with early theoretical treatments performed with soft potentials not allowing for noticeable interband transitions. ${ }^{20,22,28}$

The weight of the resonant contribution in the VB signal obtained with the $U_{2}(z)$ potential can be estimated from Eq. (3) as $1-\mathcal{P} \approx 0.8$. The steeper potential inside the solid would increase the weight of the resonant process and so decrease $\Delta$. However, the purpose of the present work is not fitting the experimental data, but revealing the robust physical mechanisms determining the measured relative time delay. For $\Omega_{X}$ above $140 \mathrm{eV}$ the resonant excitation proceeds via the (0):(3G) transition. It requires the $3 G$ reciprocal lattice vector exchange so that the dipole transition matrix elements are reduced. Consequently, the weight of the volume emission with respect to the surface one is reduced. The relative delay increases to $\Delta \approx 30$ as consistent with simple model given by Eq. (3).

The XUV energy region between 125 and $140 \mathrm{eV}$ corresponds to the resonance close to the Brillouin zone boundary. This specific case is analyzed with the help of Fig. 4. Panel (a) shows the signal on the detector placed in front of the $\operatorname{Mg}(0001)$ slab as a function of the energy of the initial occupied state which is photoexcited. (For details see Fig. 2 and related discussion.) Calculation is performed for $\Omega_{X}=$ $130 \mathrm{eV}, \gamma(z)=0$, and no NIR field. The two resonances across the projected band gap in the initial state correspond to $(0):(-2 G)$ and $(G):(-2 G)$ transitions. Close to the band gap, the initial state is repelled from the surface into the metal showing the $|\sin (\pi z n / L)|^{2}$ dependence with $L$ being the slab thickness, and $n=1,2,3, \ldots .{ }^{34}$ This is particularly well observed for the upper resonance. When the MFP effect is included in the calculations, only the electrons ejected close to the surface can escape into vacuum. The weight of the nonresonant surface emission increases and the upper resonance is suppressed. Finally, in panel (c) we show the relative delay between the emission from the LS $2 p$ and from different occupied VB states. Results are shown as function of the energy of the latter, and have been calculated from the individual streaking curves for each initial state. The 
nonresonant surface emission at low energies appears 80 as before the LS. As to the resonance contribution, it can be retarded from the LS by up to 260 as. Thus, the initial-state localization inside the metal results in substantial delay in the electron arrival on the detector at resonance ${ }^{23}$ as can be also perceived in panel (b). This is not the mean-free path, but the initial-state effect, so Eq. (3) does not apply. The final result for $\Delta$ is basically the sum of the data in Fig. 4(c) weighted by the respective contribution of the initial states to photoemission current [Fig. 4(b)]. Interestingly, $\Delta$ is as small as 9 as for $\Omega_{X}=130 \mathrm{eV}$ showing compensation of the large numbers in present case.

In conclusion, the present study reveals the role of nonresonant and resonant processes in forming the delay observed in attosecond time-resolved photoemission from LS and VB states of a metal. We have shown that the nonresonant VB electron ejection at the surface is always present. It produces electrons which come well before the LS electrons. Along with surface emission, the periodic structure of the potentials inside the metal bulk leads to the VB electron ejection via resonant interband transition. Corresponding electrons that reach the surface are excited within the distance range given by the inelastic MFP. Their arrival at the surface can be retarded or advanced with respect to LS electrons. The overall time delay between VB and LS states obtained in the atto-streaking experiment is given by the interplay between surface and resonant VB emission. Thus, for $\mathrm{W}(110)$ the VB electrons advance the LS electrons by $\approx 100$ as as measured in Ref. 12 at $\Omega_{X} \approx 90 \mathrm{eV}$. This appears consistent with the strong surface-state contribution to the VB photoemission at $100 \mathrm{eV}$ photon energies. $^{24,35}$ If the resonant process is dominating, the VB/LS delay can be much smaller, in the 10-20 as range, as calculated here for $\mathrm{Mg}(0001)$ in accordance with the experimental observations. ${ }^{13}$ In addition, we have shown that a very particular situation with large and rapidly varying relative VB/LS delay can be encountered for the XUV energies when the resonance is close to Brillouin zone boundary of the projected band structure.

P.M.E. acknowledges partial support from Basque Departamento de Educatión, Universidades, e Investigatión (Grant No. IT-366-07), CONSOLIDER, and the Spanish Ministerio de Ciencia e Innovación (Grant No. FIS2010-19609-C02-00).
${ }^{1}$ M. Hentschel et al., Nature (London) 414, 509 (2001).

${ }^{2}$ B. Bergues et al., Nat. Commun. 3, 813 (2012).

${ }^{3}$ S. Haessler et al., Nat. Phys. 6, 200 (2010).

${ }^{4}$ G. Sansone et al., Nature (London) 465, 763 (2010).

${ }^{5}$ J. Itatani, F. Quere, G. L. Yudin, M. Y. Ivanov, F. Krausz, and P. B. Corkum, Phys. Rev. Lett. 88, 173903 (2002).

${ }^{6}$ M. Kitzler, N. Milosevic, A. Scrinzi, F. Krausz, and T. Brabec, Phys. Rev. Lett. 88, 173904 (2002).

${ }^{7}$ R. Kienberger et al., Science 297, 1144 (2002).

${ }^{8}$ M. Drescher et al., Nature (London) 419, 803 (2002).

${ }^{9}$ F. Krausz and M. Ivanov, Rev. Mod. Phys. 81, 163 (2009).

${ }^{10}$ M. F. Kling and M. J. J. Vrakking, Annu. Rev. Phys. Chem. 59, 463 (2008).

${ }^{11}$ M. Schultze et al., Science 328, 1658 (2010).

${ }^{12}$ A. L. Cavalieri et al., Nature (London) 449, 1029 (2007).

${ }^{13}$ S. Neppl, R. Ernstorfer, E. M. Bothschafter, A. L. Cavalieri, D. Menzel, J. V. Barth, F. Krausz, R. Kienberger, and P. Feulner, Phys. Rev. Lett. 109, 087401 (2012).

${ }^{14}$ A. G. Borisov, D. Sánchez-Portal, R. Diéz Muiño, and P. M. Echenique, Chem. Phys. Lett. 387, 95 (2004).

${ }^{15}$ R. Díez Muiño, D. Sánchez-Portal, V. M. Silkin, E. V. Chulkov, and P. M. Echenique, Proc. Natl. Acad. Sci. USA 108, 971 (2011).

${ }^{16}$ V. Senz et al., Phys. Rev. Lett. 102, 138303 (2009).

${ }^{17}$ M. I. Stockman, M. F. Kling, U. Kleineberg, and F. Krausz, Nat. Photonics 1, 539 (2007).

${ }^{18}$ F. Süßmann and M. F. Kling, Phys. Rev. B 84, 121406 (2011).
${ }^{19}$ F. Kelkensberg, A. F. Koenderink, and M. J. J. Vrakking, New J. Phys. 14, 093034 (2012).

${ }^{20}$ A. K. Kazansky and P. M. Echenique, Phys. Rev. Lett. 102, 177401 (2009).

${ }^{21}$ C. Lemell, B. Solleder, K. Tőkési, and J. Burgdörfer, Phys. Rev. A 79, 062901 (2009).

${ }^{22}$ C.-H. Zhang and U. Thumm, Phys. Rev. Lett. 102, 123601 (2009).

${ }^{23}$ E. E. Krasovskii, Phys. Rev. B 84, 195106 (2011).

${ }^{24}$ A. G. Rybkin, E. E. Krasovskii, D. Marchenko, E. V. Chulkov, A. Varykhalov, O. Rader, and A. M. Shikin, Phys. Rev. B 86, 035117 (2012)

${ }^{25}$ R. A. Bartynski, R. H. Gaylord, T. Gustafsson, and E. W. Plummer, Phys. Rev. B 33, 3644 (1986).

${ }^{26}$ L. Aballe, C. Rogero, and K. Horn, Surf. Sci. 518, 141 (2002).

${ }^{27}$ F. Schiller, M. Heber, V. D. P. Servedio, and C. Laubschat, Phys. Rev. B 70, 125106 (2004).

${ }^{28}$ J. C. Baggesen and L. B. Madsen, Phys. Rev. A 78, 032903 (2008).

${ }^{29}$ A. Liebsch, Electronic Excitations at Metal Surfaces, (Plenum Press, New York, 1997).

${ }^{30}$ Solid-State Photoemission and Related Methods, edited by W. Schattke and M. A. Van Hove (Wiley-VCH, Weinheim, 2003).

${ }^{31}$ C. Leforestier et al., J. Comput. Phys. 94, 59 (1991).

${ }^{32}$ E. V. Chulkov, V. M. Silkin, and P. M. Echenique, Surf. Sci. 437, 330 (1999).

${ }^{33}$ C. J. Powell and A. Jablonski, J. Phys. Chem. Ref. Data 28, 19 (1999).

${ }^{34}$ T.-C. Chiang, Surf. Sci. Rep. 39, 181 (2000).

${ }^{35}$ K. Miyamoto et al., Phys. Rev. Lett. 108, 066808 (2012). 